\title{
Preparation and Physical Properties of Red-photoluminescent Graphene/Europium(III)/Picolinate
}

\author{
Jang Yong Kim, Jin Bong Lee, Hye Jin Kim, Koo Shin, ${ }^{\dagger}$ Yun-Sik Yu, and Yunghee Oh* \\ Convergence of IT Devices Institute Busan, Dong-Eui University, Pusan 614-714, Korea. *E-mail: yhoh@deu.ac.kr \\ ${ }^{\dagger}$ Department of Chemistry, Sejong University, Seoul 143-747, Korea \\ Received February 26, 2010, Accepted April 1, 2010
}

\begin{abstract}
Novel photoluminescent graphene, graphene/europium/picolinate, was made from the reaction of the graphene carboxylate, europium(III) and $\alpha$-pyridoin anion in methanol solution. There was evidence on IR spectroscopic data that europium metal was coordinated by graphene carboxylate. The graphene/europium/picolinate materials emitted the red luminescence with main peaks at 609 and $695 \mathrm{~nm}$, when solid sample was excited by an UV light at the wavelength of $254 \mathrm{~nm}$. The surface morphology and physical properties of the graphene/europium/picolinate product have been investigated by IR, PL, SEM, TEM and TGA measurements.
\end{abstract}

Key Words: Europium, Picolinate, Pyridoin, Graphene, Photoluminescent

\section{Introduction}

Recently graphene has attracted great interest in many research fields. Graphene is defined as a flat carbon monolayered sheet made originally from graphite. The graphene sheet is manually made from the peeling of graphite using scotch tape, or made from the epitaxial growth. Another method to get graphene on a large scale can be done via chemical reactions in solution. ${ }^{1-3}$ When commercially available graphite is treated under strong oxidizing acidic conditions, graphite oxides or carboxylic acids are produced. ${ }^{45}$ The functional groups are attached at the edges of the plane and make the graphite stacks exfoliated, followed by the $\pi$-stacked carbon layer separating into nanostructured monolayer flat sheet, so-called graphene. The graphene carboxylic acid is used for production of new materials. With graphene's characteristics such as the nanostructural property, electrical conducting and its high mechanical strength, ${ }^{6}$ the combination of graphene and metal has a great potential for industrial usage and research fields. Doping or intercalating metal into graphene has been studied because they exhibit unique properties. ${ }^{7-9}$

We are interested in making photoluminescent graphene containing lanthanide metal, which is chemically bonded between graphene carboxylate anion, metal and other small ligands. Europium and $\alpha$-pyridoin are good candidates for this purpose; when these react, the photoluminescent product is yielded immediately. We have reported that when europium(III) reacted with the $\alpha$-pyridoin anion in methanol aerobically, metal complex in which europium was coordinated by picolinates (pic) was produced as shown in Figure $1 .^{10}$ The complex exhibited bright light-emitting properties when it was irradiated by UV light. We had fully characterized the crystal structure and the light-emitting properties of the europium complex.

The purposes of this paper are; (1) to prepare graphene/europium materials and to characterize chemical bonds between graphene carboxylate and metal ion, (2) to prepare a photoluminescent graphene/europium material by using $\alpha$-pyridoin anion and (3) to characterize physical properties of the materials in- a-pyridoin $/ \mathrm{OH}^{-}+\mathrm{Eu}\left(\mathrm{NO}_{3}\right)_{3} \cdot 5 \mathrm{H}_{2} \mathrm{O} \stackrel{[\mathrm{O}]}{\longrightarrow} \mathrm{Na}\left[\mathrm{Eu}(\text { pic })_{4}\right] \cdot 2 \mathrm{H}_{2} \mathrm{O}$
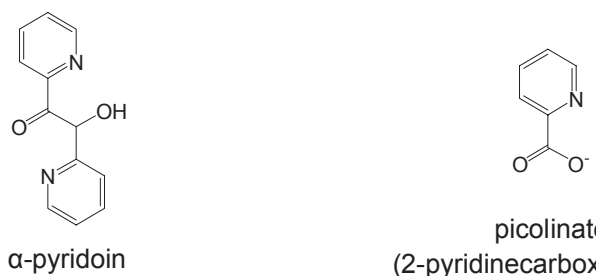

picolinate

(2-pyridinecarboxylate, pic)

Figure 1. Reaction of europium(III) ion and $\alpha$-pyridoin anion.

cluding light-emitting properties and surface morphology. There have been previous reports on metal graphite intercalation compound via ignition in the furnace. ${ }^{11}$ However, this is the first report on photoluminescent graphene/lanthanide made from the mild reaction condition.

\section{Experimental}

Preparation. Graphene carboxylic acid was prepared by reported methods in the literature. ${ }^{5}$ Graphene/europium materials were prepared by the following procedure. The graphene carboxylic acid $(0.5 \mathrm{~g})$, water $(20 \mathrm{~mL})$ and $\mathrm{NaOH}(1 \mathrm{M}$ aqueous solution, $1 \mathrm{~mL}$ ) were sonicated for 30 minutes in a Cole-Parmer sonicator at a power lever of $200 \mathrm{~W}$. Europium nitrate, $\mathrm{Eu}\left(\mathrm{NO}_{3}\right)_{3}$. $5 \mathrm{H}_{2} \mathrm{O}(0.428 \mathrm{~g}, 1 \mathrm{mmol})$ was added and stirred overnight. The solids were centrifuged, washed with water and acetone. The graphene/europium solids were dried in a vacuum oven overnight.

Photoluminescent graphene/europium/picolinate materials were prepared by the following procedure. The $\alpha$-pyridoin $(0.420 \mathrm{~g}, 2 \mathrm{mmol})$ was dissolved in methanol $(20 \mathrm{~mL})$, and $\mathrm{NaOH}$ (1 M aqueous solution, $2 \mathrm{~mL}$ ) was added. The graphene/ europium solids and methanol $(20 \mathrm{~mL})$ were sonicated for 30 minutes followed by adding the $\alpha$-pyridoin anion methanol solution. The reaction mixture was stirred overnight. Solids were 


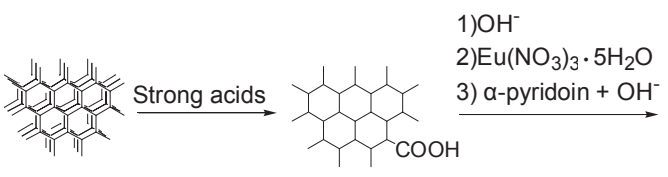

(a) (b)

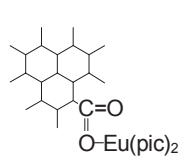

(c)
Figure 2. Schematic illustration of graphene materials preparation: (a) graphite, (b) graphene carboxylic acid and (c) graphene/europium/ picolinate.

centrifuged and washed with methanol several times to remove residual $\alpha$-pyridoin and/or europium compounds detached from graphene. The solids were then dried under vacuum. The simplified reaction scheme and proposed structure of the graphene/ europium/picolinate materials were shown in Figure 2.

Characterization. IR spectra were recorded on FT/IR-300E spectrophotometer with $\mathrm{KBr}$ pellet sample. Photoluminescent emission spectra were recorded on a DASA-5000 spectrophotometer equipped with a $150 \mathrm{~W}$ Xenon lamp as the excitation source. The sample for the PL measurement was prepared by pressing solids into a pellet when pumping out air. SEM micrographs were obtained by using a JSM-35CF Scanning Electron Microscope. TEM images and selected area electron diffraction pic-

(a)

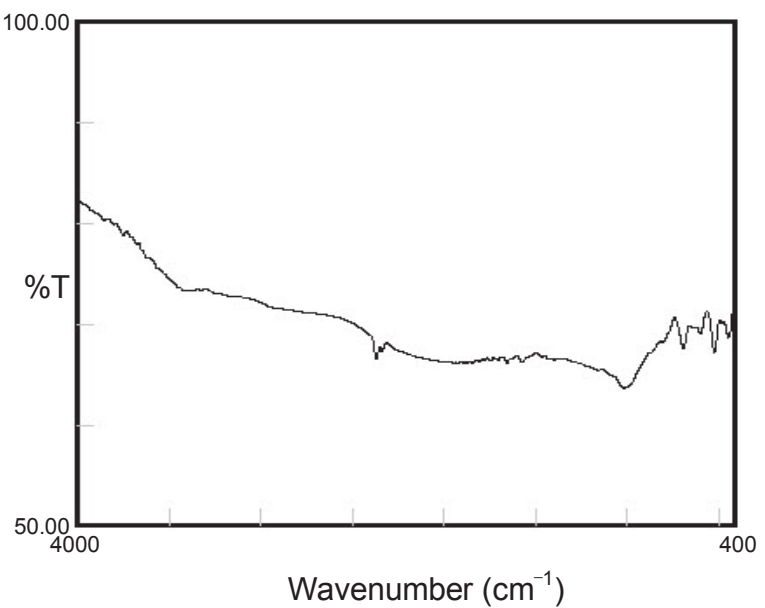

(c)

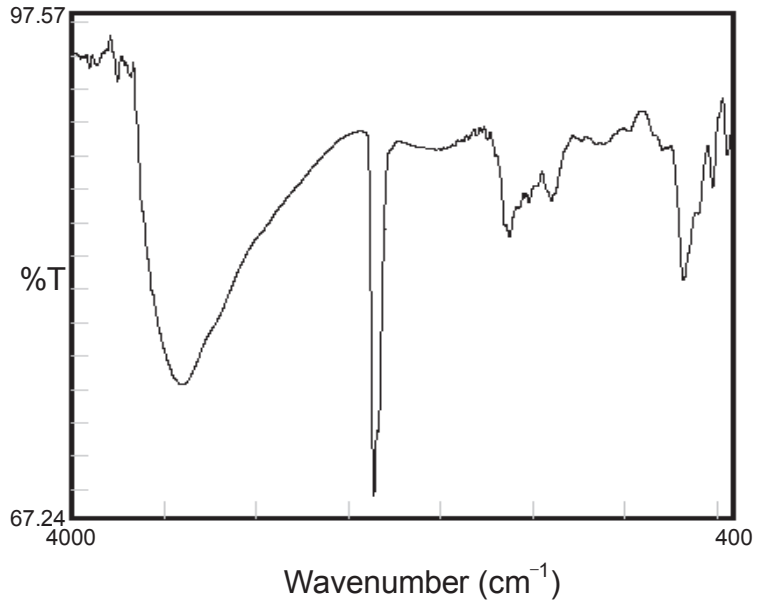

tures were recorded on a Jeol 2010 transmission electron microscope, using an accelerating voltage of $200 \mathrm{kV}$. The samples for TEM were prepared by dipping-drying solid/methanol dispersion onto a copper disk. TGA analyses were carried out up to $1000{ }^{\circ} \mathrm{C}$ at the temperature rate of $5{ }^{\circ} \mathrm{C}$ by using a DTG-50H instrument.

\section{Results and Discussion}

The FT-IR spectra of graphite (a), graphene carboxylic acid (b), graphene/europium (c) and ghraphene/europium with picolinate ligand, (graphene/europium(pic)) (d) are shown in Figure 3. The changes of the $\mathrm{COOH}$ functional group bands positions from graphene carboxylic acid to graphene/europium(III) are evident in IR spectra. The bands of $v_{\mathrm{C}=\mathrm{O}}$ in $\mathrm{COOH}$ of the graphene carboxylic acid are observed at 1720 and $1621 \mathrm{~cm}^{-1}$, as shown in the (b). These are typical values in carboxylated graphenes. ${ }^{4,12-16}$ However, the bands of $v_{\mathrm{C}=0}$ in $\mathrm{COO}^{-}$of europium bonded graphene appeared at around 1650 and $1400 \mathrm{~cm}^{-1}$, as shown in (c) and (d), indicating that the oxygen atoms of carboxylate ion coordinate metal ions. ${ }^{17}$

The PL spectrum of the graphene/europium(pic) is shown in Figure 4, which contains the same main band positions as the corresponding europium(pic) complex, with different widths.

(b)

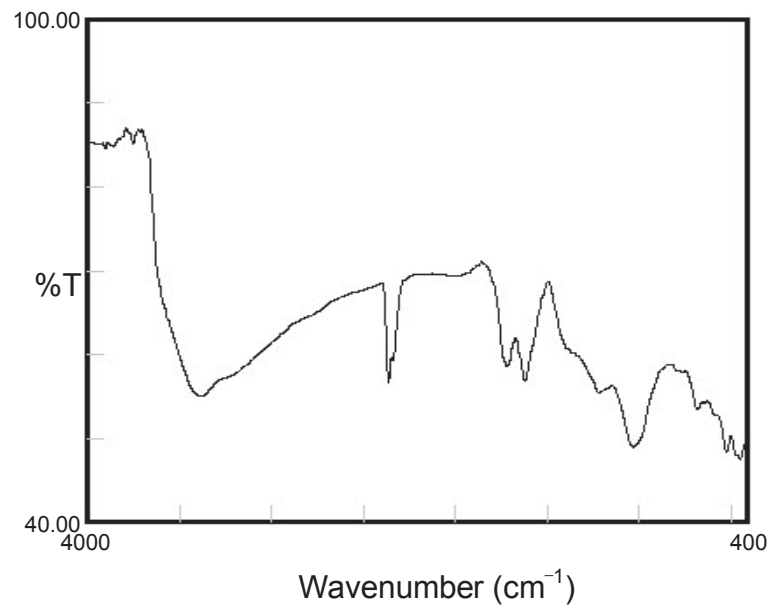

(d)

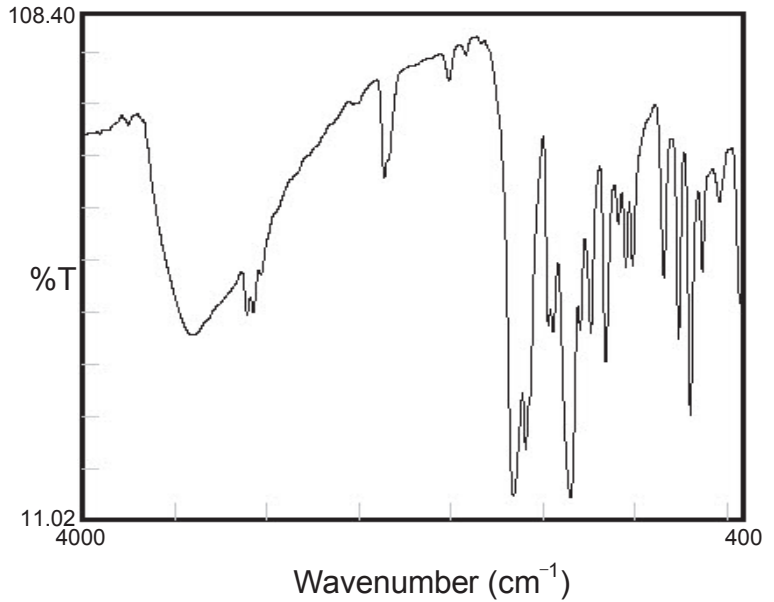

Figure 3. FT-IR Spectra of (a) graphite (b) graphene carboxylic acid (c) graphene/europium (d) graphene/europium(pic). 
(a)

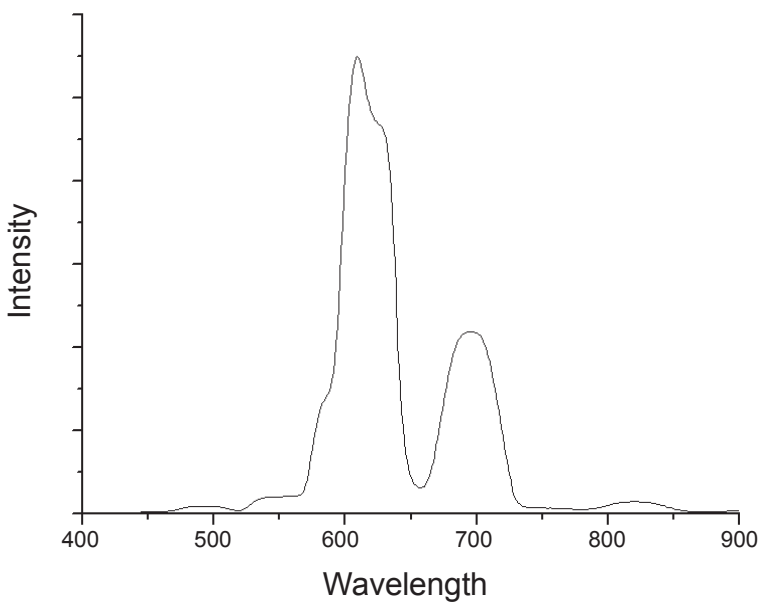

(b)

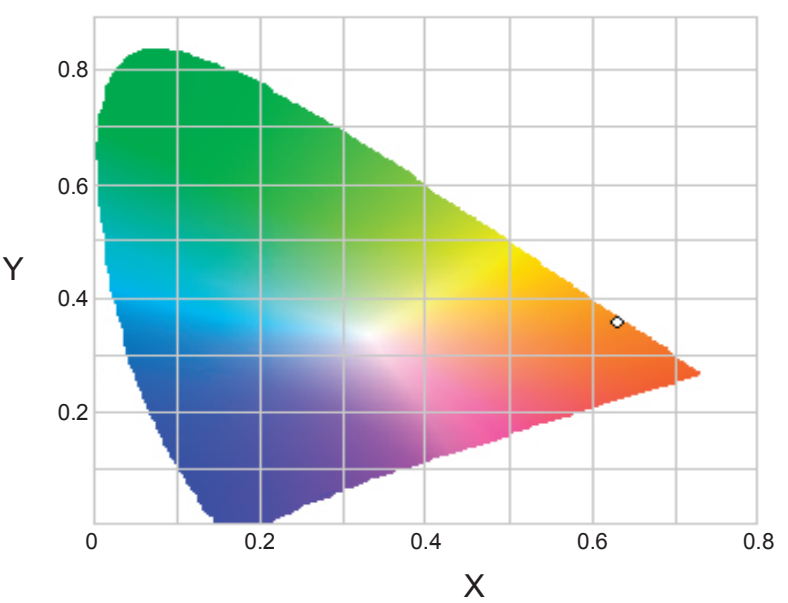

Figure 4. PL spectrum of graphene/europium(pic) in solid state in pellet ( $\left.\lambda_{\mathrm{exc}}=254 \mathrm{~nm}\right)(\mathrm{a})$ and its CIE coordinates (b).
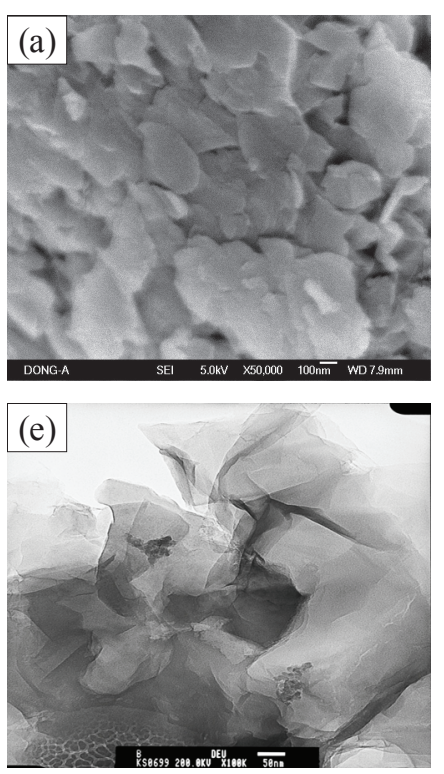
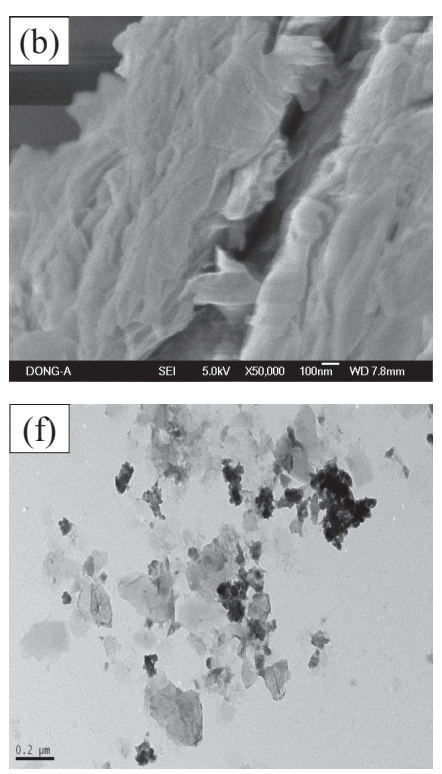
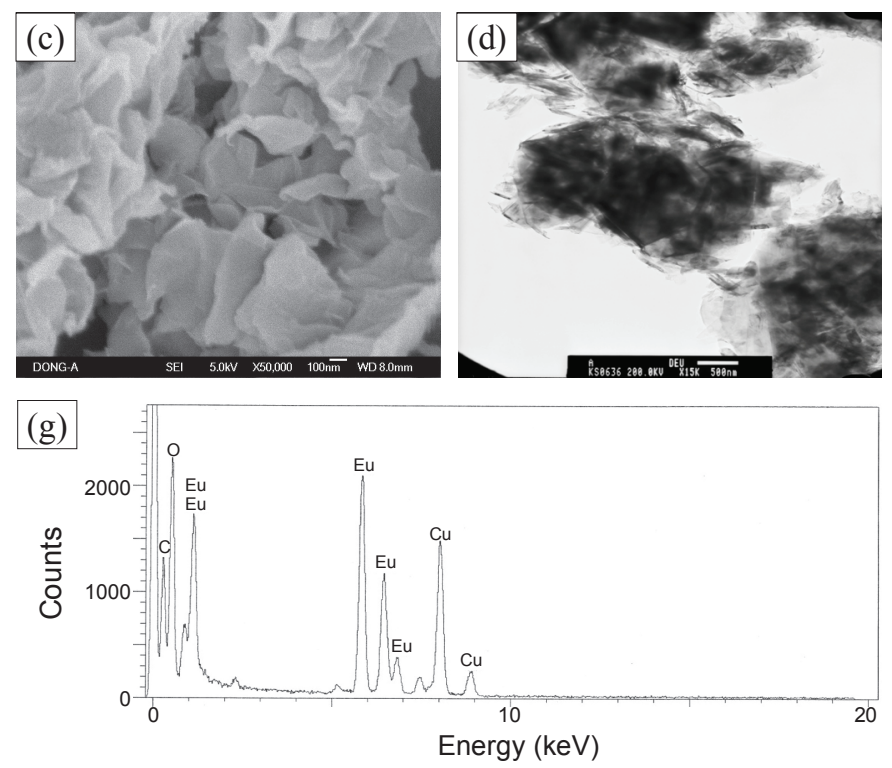

Figure 5. SEM (a, b, c), TEM (d, e, f) pictures and EDS analysis (g): (a,d) graphite, (b,e) graphene carboxylic acid, (c,f) graphene/europium(pic) and (g) EDS analysis of the black spots in (f) image.

Graphene/europium(pic) has a broader width than the corresponding europium(pic) complex. Due to the unavailability of Graphene/europium(pic) in a solution sample, the different band widths outcome are presumably caused by measuring the graphene/europium(pic) sample as a solid pellet, while europium (pic) complex was measured in solution. ${ }^{10}$ The graphene/europium(pic) strongly emitted red light at 587(sh), 609, 630(sh) and $695 \mathrm{~nm}$ when it was excited by a UV light of $254 \mathrm{~nm}$ wavelength. Those four ${ }^{5} \mathrm{D}_{0} \rightarrow{ }^{7} \mathrm{~F}$ peaks are consistent with other europium complexes. ${ }^{18}$ The observed bands are not well-resolved in solid pellet sample compared to the solution sample of the corresponding europium picolinate complex, ${ }^{10}$ but band positions are consistent. The emission color was analyzed by using the Commision de I Enclairage (CIE) chromaticity coordinate diagram as shown in Figure 4 (b). The purity of the red color is $98.6 \%$ with $\mathrm{x}=0.64$ and $\mathrm{y}=0.36$ in color coordinates.

The SEM and TEM images and EDS analysis are shown in
Figure 5. Compared to the graphite, graphene and graphene/europium (pic) images are clearly distinguished by exfoliated sheet type in TEM images. Black spots in the TEM picture of the graphene/europium(pic) were europium metals, which was confirmed by the EDS analysis of the spots.

The thermogravimetric analysis shows graphite, the oxidized graphene and graphene/europium(pic) oxidizing in dry air at $600{ }^{\circ} \mathrm{C}-800^{\circ} \mathrm{C}$, as shown in Figure 6. The results coincide with other reports. ${ }^{4}$ Substantial weight loss $(10 \%)$ due to the carboxylic group is observed at $200{ }^{\circ} \mathrm{C}$ in Figure 6 (b). Graphene carboxylic acid was used to control the moles of $\mathrm{NaOH}$ in order to make the mole ratio of graphene carboxylate to europium 1 to 1 . The $400{ }^{\circ} \mathrm{C}$ weight loss of the graphene/europium(pic) came from decomposition of the europium picolinate.

Initially, we expected that the graphene/europium(pic) would show conductivities at a level that would require electronic materials, but the product did not exhibit any conductivities in our 


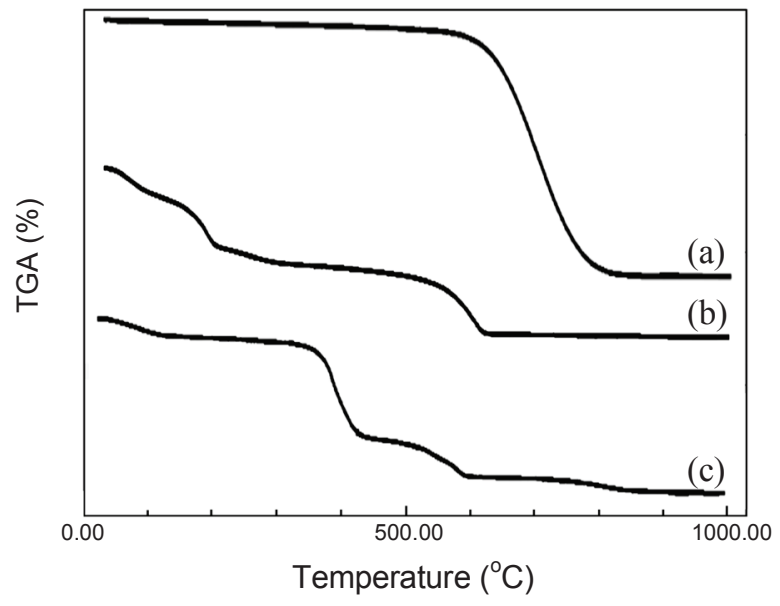

Figure 6. TGA data under dry air with a temperature ramp rate of $5{ }^{\circ} \mathrm{C} /$ min : (a) graphite, (b) graphene carboxylic acid and (c) graphene/europium(pic).

reaction condition. Further research to improve the conductivities by controlling the graphene contents is an ongoing process.

\section{Conclusions}

We prepared graphene/europium materials, a form of graphene carboxylate, coordinated to europium(III), which was approved by IR spectroscopic data. Novel photoluminescent graphene/europium/picolinate materials were prepared from the graphene/europium and $\alpha$-pyridoin anion in methanol solution. The product strongly emitted red light at $587(\mathrm{sh}), 609,630(\mathrm{sh})$ and $695 \mathrm{~nm}$ when excited by a UV light at the wavelength of $254 \mathrm{~nm}$. SEM and TEM images and TGA analysis are consistent from well-stacked graphite to exfoliated graphene/europium/picolinate materials with europium existence.
Acknowledgments. This work was supported in part by MKE (IITA), Busan Metropolitan City and Dong-Eui University (08GIBAN-13, Convergence of IT Devices Institute Busan).

\section{References}

1. Geng, Y.; Wang, S. J.; Kim, J. K. J. Coll. Inter. Sci. 2009, 336, 592.

2. Novoselov, K. S.; Geim, A. K.; Morozev, S. V.; Jiang, D.; Zhang, Y.; Dubonos, S. V.; Grigorieva, I. V.; Firsov, A. A. Science 2004, $306,666$.

3. Arsat, R.; Breedon, M.; Shafiei, M.; Spizziri, S. P.; Gilje, S.; Kaner, R. B. et al. Chem. Phys. Lett. 2009, 467, 344.

4. Niyogi, S.; Bekyarova, E.; Itkis, M. E.; McWilliams, J. L.; Hamon, M. A.; Haddon, R. C. J. Am. Chem. Soc. 2006, 128, 7720.

5. Gilje, S.; Han, S.; Wang, M.; Wang, K. L.; Kaner, R. B. Nano Lett. 2007, 7, 3394.

6. Stoyanov, S. R.; Titov, A. V.; Kral, P. Coord. Chem. Rev. 2009, 253,2852

7. Yang, Y.; Wang, H.; Lu, X.; Zhao, Y.; Li, X.; Wang, C. Mater. Science Engineer B 2007, 140, 48.

8. Wang, W. W.; Li, X. Y.; Li, H. N. Phy. Lett. A 2008, 372, 6677.

9. Cahen, S.; Vangelisti, R. J. Phys. Chem. Solids 2006, 67, 1223.

10. Hong, J. H.; Oh, Y.; Kim, Y. M.; Kang, S. K.; Choi, J. K.; Kim, W. S. et al. Crys. Growth Design 2008, 8, 1364.

11. Cahen, S.; Vangelisti, R.; Bellouard, C. Carbon 2006, 44, 259.

12. Wang, H.; Hao, Q.; Yang, X.; Lu, L.; Wang, X. Electrochem. Comm. 2009, 11, 1158.

13. Wu, T. M.; Lin, Y. W. Polymer 2006, 47, 3576.

14. Yuen, S. M.; Ma, C. C. M.; Lin, Y. Y.; Kuan, H. C. Composit. Science and Technol. 2007, 67, 2564.

15. Zhu, B. K.; Xie, S. H.; Xu, Z. K.; Xu, Y. Y. Composit. Science and Technol. 2006, 66, 548.

16. Du, X. S.; Xiao, M.; Meng, Y. Z. Eur. Polymer J. 2004, 40, 1489.

17. Tian, L.; Ren, N.; Zhang, J. J.; Liu, H. M.; Bai, J. H.; Ye, H. M. Inorg. Chim. Acta 2009, 362, 3388.

18. Kumar, R.; Makrandi, J. K.; Singh, I.; Khatkar, S. P. J. Luminescence 2008, 126(8), 1297. 